\title{
Uterine Artery Doppler Velocimetry in Placenta Previa and Placenta Accreta
}

\author{
MOHAMED A.M. BOSTAN, M.Sc.; HESHAM M. EL-TOKHY, M.D.; RASHA A. EL-SHAFAY, M.D. and \\ NAREMAN M. EL-HAMAMY, M.D.
}

The Department of Obstetrics and Gynecology, Faculty of Medicine, Tanta University

\begin{abstract}
Background: Placenta previa and placenta accreta are common obstetrical problem. Placenta previa is partially or totally implanted placenta in the lower uterine segment, this abnormality in the site occurs with different degrees, placenta previa lateralis, marginalis and centralis either incomplete or complete. Abnormal placental adhesion means that the placenta invades the uterine myometrium with different degree. This morbid adhesion is called "Placenta Accreta", with different grades according to the degree of the trophoblastic invasion through the myometrium and bladder serosa as placenta accreta vera, placenta increta and placenta percreta. Incidence of placenta accreta is increased over the past years mainly due to increasing rates of cesarean delivery. Placenta accreta may lead to massive obstetric hemorrhage with attempt to remove the placenta leading to several complications up to death. Diagnosis of placenta accreta can be done by different modalities such as Ultrasound Grey scale, Color Doppler, Magnetic Resonance Imaging (MRI). Ultrasonography is usually employed as the primary modality for antenatal diagnosis of invasive placentation. Over recent years, Color Doppler Sonography (CDS) have been started to be used to predict placental abnormalities. CDS, a noninvasive and simple tool, is useful indistinguishing normal and abnormal placentation.
\end{abstract}

Aim of Study: The aim of this work was to study uterine artery doppler velocimeteric changes in patients with placenta previa and placenta accrete.

Patients and Methods: 30 pregnant females were included in this comparative prospective clinical study subdivided into two group; Group (A) Includes 15 patients with anterior placenta previa, and Group (B) Include 15 patients with anterior placenta previa accrete. Both groups were diagnosed by trans-abdominal Ultrasonography either from outpatient clinics or inpatient department of Tanta University Hospital seeking for antenatal care from Octorber 2017 to August 2018 All patients underwent uterine artery Doppler velocimetry to measure the mean Resistive Index (RI) and Pulsatility Index (PI) in the third trimester and compared between both groups.

Correspondence to: Dr. Mohamed A.M. Bostan, The Department of Obstetrics and Gynecology, Faculty of Medicine, Tanta University
Results: the mean uterine artery velocemtric changes including mean Resistive Indices (RI) and mean Pusitility Indices (PI) were significantly lower in the placenta accreta group compared to placenta previa group (with mean for RI 0.41 versus $0.45 ; p=0.001$ ) and (with mean for PI 0.50 versus $0.56 ; p=0.001$ ), the uterine artery Doppler values in combination with maternal characteristics, such as previous history of cesarean deliveries, will improve screening accuracy of placenta accrete $(p=0.022)$.

Conclustion: This study suggests that the mean PI and RI measured by uterine artery Doppler velocimetry is reduced in patients with placenta accreta compared to those without accreta. Accuracy of diagnosis can be potentially improved in invasive placentation if uterine artery Doppler values and the history of cesarean delivery are combined.

Key Words: Placentaprevia-Placenta accrete-Obstetric ultrasound - Color Doppler Sonography (CDS) - Resistive Indices (RI) - Pusitility Indices (PI).

\section{Introduction}

PLACENTA is an amazing organ which is responsible for the mechanical and physiological connection between mother and her fetus [1]. It has a wide range of abnormalities, some related to the site of implantation, mostly the placenta is implanted in the upper uterine segment while in small percentage, it is implanted in the lower uterine segment, this abnormality in the site is called placenta previa with its different degrees, placenta previa lateralis, marginalis and centralis either incomplete or complete [2].

Abnormal placental adhesion means that the placenta invades the uterine myometrium with different degrees. It is due to defect in the Nitabach's layer associated with previous uterine surgery. This morbid adhesion is called "Placenta Accreta", with different grades according to the degree of the trophoblastic invasion through the myometrium and bladder serosa as placenta accreta vera, placenta increta and placenta percreta [3]. 
Incidence of placenta accreta is increased over the past 50 years mainly due to increasing rates of cesarean delivery. The incidence was cited as 1 in 2500 in the 1980s, and currently, the American College of Obstetricians and Gynecologists (2012) cites it to be as high as 1 in 533 deliveries [4]

Placenta accreta may lead to massive obstetric hemorrhage with attempt to remove the placenta leading to several complications such as disseminated intravascular coagulopathy, cesarean hysterectomy, multiple surgical injuries to the ureters, bladder, and other viscera, adult respiratory distress syndrome, renal failure, hypovolemic shock, circulatory collapse and need for postoperative ICU or death. The average amount of blood loss at delivery in a case of placenta accreta is 3,000$5,000 \mathrm{ml} \mathrm{[5].}$

Diagnosis of placenta accreta can be done by different modalities such as Ultrasound Grey scale, Color Doppler, Magnetic Resonance Imaging (MRI). Ultrasonography is usually employed as the primary modality for antenatal diagnosis of invasive placentation. Specific sonographic features of placenta accreta appear as lose of retro placental hypo echoic clear zone, loss of bladder wall uterine interphase, presence of placental lacunae (vascular spaces), and presence of hyper vascularity of interphase between the uterine serosa and bladder wall. Magnetic Resonance Imaging MRI is reported to be complementary to ultrasound, as it may help in diagnosing invasive placentation, especially in those cases in which ultrasound is not conclusive as posterior placenta previa [6].

Uterine artery Doppler studies have suggested that a high uterine artery Pulsatility Index (PI) might be an indirect sign of impaired placentation which leads to adverse pregnancy outcomes like such as preeclampsia and intrauterine growth restriction $[\mathbf{7 , 8}]$

\section{Patients and Methods}

This prospective study was conducted in Department of Obstetrics \& Gynecology Tanta University Hospitals, from Octorber 2017 to August 2018. 30 pregnant females from outpatient clinics or inpatient department of Tanta University Hospital seeking for antenatal care females they were subdivided into two groups: Group (A) included 15 patients with anterior placenta previa, and Group (B) included 15 patients with anterior placent previa accrete, both groups were diagnosed by trans-abdominal Ultrasonography, aged between 25 to 35 years old and all were in the third trimester (after 34 weeks of gestation).
The aim of this work was to study uterine artery doppler velocimeteric changes in patients with placenta previa and placenta accreta.

Exclusion criteria were medical disorders associated with the pregnancy (hypertension, diabetes mellitus, renal diseases, thyroid dysfunctions, cardiovascular disease ), multiple pregnancy, intrauterine growth restriction, congenital malformations and chromosomal abnormalities.

The study was approved by Ethical Committee at Faculty of Medicine Tanta University. All patients gave oral and written informed consent prior to the examination.

Sonography was performed by a single welltrained same observer to avoid inter observer variability using ultrasound machines (Mindray DC-30) with a multifrequency volumetric transabdominal transducer.

The uterine artery Doppler measurements were taken at their crossing with the external iliac artery from both sides of the uterus, and color Doppler imaging will be used to identify the uterine artery.

Uterine artery Doppler measurements were performed on the left and right uterine arteries, and the mean PI and Resistive Index (RI) were defined as the average of the two vessels.

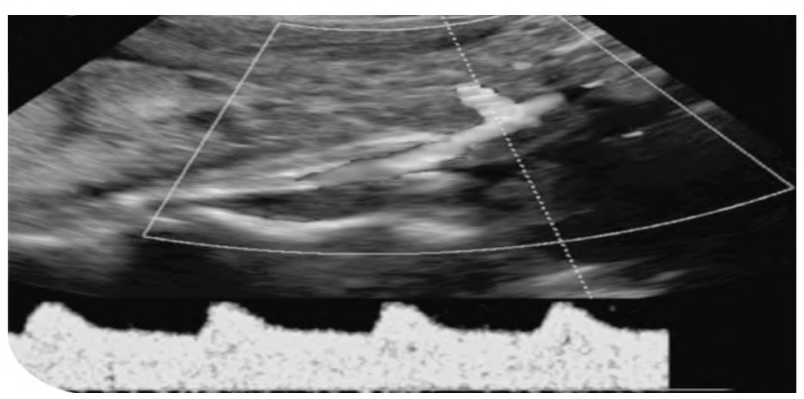

Fig. (1): (A) Normal and (B) Abnormal uterine artery Doppler flow [9]

\section{Results}

The study was carried on (30) pregnant women, (15) patients with placenta previa Group (A), and the other (15) with placenta previa accreta Group (B).

Table (1): Summarizes demographic data of the two groups.

\begin{tabular}{lllc}
\hline Item & Group (A) & Group (B) & $p$-value \\
\hline - Maternal age (years) & $30.92 \pm 3.36$ & $32.33 \pm 2.87$ & 0.114 \\
- Gestational age (weeks) & $36.57 \pm 1.22$ & $36.73 \pm 0.70$ & 0.579 \\
- Parity & $2 \pm 1.35$ & $2.33 \pm 0.72$ & 0.395 \\
- History of previous Cesarean & $1.64 \pm 1.08$ & $2.46 \pm 0.83$ & 0.022 \\
$\quad$ sections & & & \\
- History of previous abortions & $0.21 \pm 0.42$ & $0.26 \pm 0.59$ & 0.723 \\
\hline
\end{tabular}


There were significant correlation between history of previous cesarean sections and detection of placenta accrete.

Table (2): Summarizes uterine artery Doppler velocimetric changes expressed in mean resistance index and mean pulsatility index in cases with placenta previa and placenta accrete.

\begin{tabular}{llll}
\hline Item & Group (A) & Group (B) & $p$-value \\
\hline $\begin{array}{l}\text { Mean resistance index of } \\
\text { uterine artery }(R I):\end{array}$ & & & \\
$\quad$ Mean \pm SD & $0.45 \pm 0.022$ & $4 \pm 0.01$ & $<0.001$ \\
$\quad$ Range & $0.42-0.47$ & $0.39-0.45$ & \\
Mean pulsatility index of & & & \\
uterine artery $(P I):$ & & & \\
$\quad$ Mean \pm SD & $0.56 \pm 0.01$ & $0.50 \pm 0.01$ & $<0.001$ \\
$\quad$ Range & $0.53-0.59$ & $0.49-0.52$ & \\
\hline
\end{tabular}

In comparision between the two study groups, as regards the mean resistive and pulsatility indices of uterine arteries, these indices were statistically significant lower in Group B than Group A.

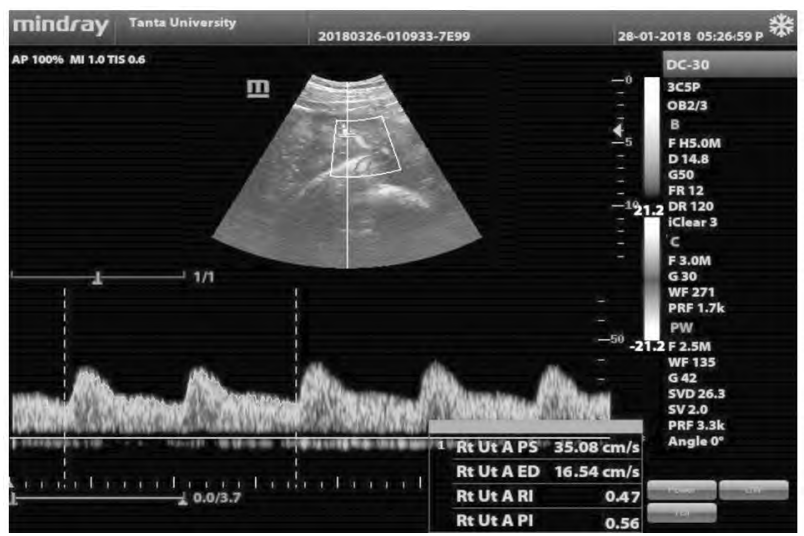

Fig. (2): Mean resitance Index (RI) and mean Pulsitility Index (PI) of right uterine artery artery Doppler in a case with placenta previa.

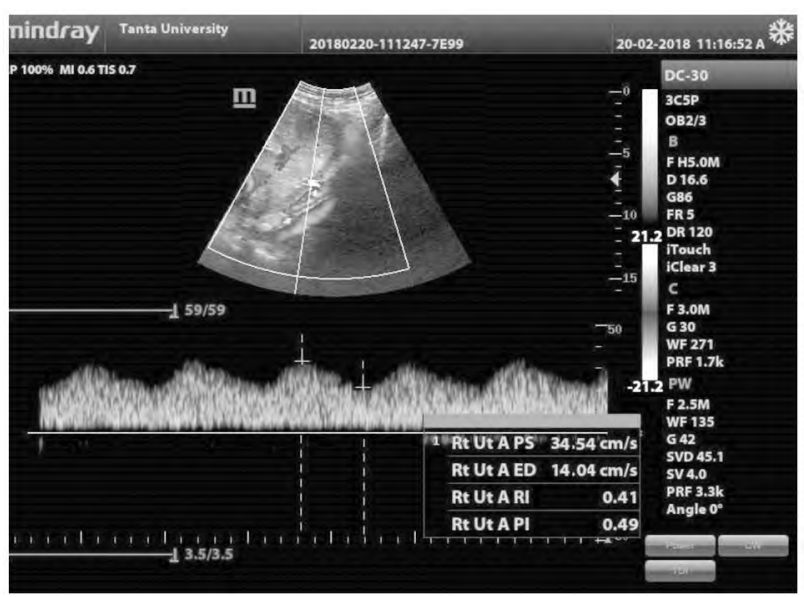

Fig. (3): Mean Resitance Index (RI) and mean Pulsitility Index of (PI) right uterine artery artery Doppler in a case with placenta accrete.

\section{Discussion}

Placenta accreta is a significant cause of maternal morbidity and mortality and is presently the most common reason for emergency postpartum hysterectomy [10]

In this study we investigated 30 pregnant women with history of one or more previous cesarean sections, after 34 weeks of gestation, 15 patients with placenta previa anterior, and the other 15 with placent previa accreta anterior diagnosed by transabdominal Ultrasonography either from outpatient clinics or inpatient department of Tanta University Hospital seeking for antenatal care and uterine artery Doppler velocimetric measurements and indices were recorded.

First, our study findings showed that the mean uterine artery pulsetility and resistive indices were decreased in patients with placenta accreta than placenta previa. Second, maternal characteristics, such as previous history of cesarean deliveries, were statistically significant between both groups.

Although we were able to demonstrate that uterine artery Doppler velocimetric values may be affected by the presence or absence of abnormal placental adherence but failed to demonstrate a single powerful pathognomonic factor for placenta accreta. Thus, we combined the history of cesarean delivery and uterine artery Doppler measurements for placenta accreta screening.

According to Cho H.Y. et al., [11], a total of 11,210 cases were evaluated, including 403 cases of placenta previa and 39 cases of (placenta accreta). Clinical records of all deliveries between April 1991 and March 2013 were retrospectively analyzed all patients underwent uterine artery Doppler velocimetry to measure the mean resistive and pulsatility indices in the third trimester. And this study found that the mean uterine artery PI was significantly lower in the placenta accreta group compared to previa alone ( 0.51 versus $0.57 ; p=.002)$. The odds ratios for placenta accreta were 2.4 for 2 or more previous abortions $(p=.011)$ and 5.3 and 7.0 for 1 and 2 or more previous cesarean deliveries $(p=.001$ and .005). With an increase in the mean PI by 0.01 , the odds ratio for placenta accreta decreased by $0.94(p<.001)$. The diagnostic accuracy of placenta accreta can be potentially improved if uterine artery Doppler values and the history of cesarean delivery are combined.

According to Zamanskaya et al., [12], fifty pregnant women with normal placentation (Group 1), 50 women with placenta previa (Group 2) and 
28 women with placenta accreta (Group 3) were examined in their study. The ultrasound examination included the traditional fetometry and an assessment of fetal anatomy and localization of the placenta. In addition, the Doppler ultrasound was used to examine the blood flow in the uterine arteries as well as the umbilical artery and the middle cerebral artery of the fetus. The measurements were performed on the 20-22 nd, 30-32nd and 35-36th weeks of gestation. The pulsatility index in the right uterine artery was significantly lower in patients with placenta accreta as compared with the two other groups. The data on fetoplacental hemodynamics indicated differences in the umbilical artery blood flow between women with placenta accreta, placenta previa and normal placental localization on the 20-22 nd and 35-36 th weeks of gestation. Along with the above findings in the uterine and fetoplacental hemodynamics, the parameters of blood flow in the fetal middle cerebral artery did not differ significantly between the three groups of women at any period of gestation. So The conditions of placenta accreta and placenta previa are associated with a decrease in the peripheral vascular resistance in both uterine arteries during all periods of gestation. This is supported by the lower values of the pulsatility index in these blood vessels in comparison to the values in patients with normal placental localization.

According to Usta et al., [13], patients with placenta previa $(n=347)$ delivered over 20 years were reviewed, divided into placenta accreta (cases, $n=22$ ) and no accreta (controls, $n=325$ ), and compared to identify risk factors and complications of placenta previa-accreta and found out that PA incidence increased with the number of previous CS: $1.9 \%, 15.6 \%, 23.5 \%, 29.4 \%, 33.3 \%$, and $50.0 \%$ after $0,1,2,3,4$, and 5 previous $\mathrm{CS}$, respectively.

\section{Conclusion:}

The mean uterine artery velocemtric changes including mean Resistive Indices (RI) and mean Pusitility Indices (PI) were significantly lower in the placenta accreta group compared to placenta previa group. However, the uterine artery Doppler values alone might be insufficient for differentiating placenta accreta from placenta previa, but a combination with maternal characteristics, such as previous history of cesarean deliveries, will improve screening accuracy of placenta accreta.

\section{Acknowledgments:}

This research was carried out without funding. Great thanks to Obstetrics and Gynecology Department Faculty of Medecine Tanta University.

\section{Conflicts of interest:}

No conflicts of interest declared.

\section{Authors' contributions:}

All authors had equal role in design, work, statistical analysis and manuscript writing. All authors have approved the final article work.

\section{References}

1- KONKEL L.: The Role of the Placenta in Fetal Programming. Environ Health Perspect, 124 (7): 124-9, 2016.

2- CUNNINGHAM F. GARY: Placental abnormalities. Williams Obstetrics. (24 th edition), 116-19, 2018.

3- BELFORT M.A.: Placenta accreta. Am. J. Obstet. Gynecol., 203 (5): 430-9, 2010.

4- WU S., KOCHERGINSKY M. and HIBBARD J.U.: Abnormal placentation: Twenty-year analysis. Am. J. Obstet. Gynecol., 192 (5): 1458-61, 2005.

5- KASTNER E.S., FIGUEROA R., GARRY D. and MAU LIK D.: Emergency peripartum hysterectomy: Experience at a community teaching hospital. Obstet. Gynecol., 99 (6): 971-5, 2002.

6- TONNI G. and ARAUJO JUNIOR E.: Three-dimensional ultrasound in obstetrics practice: Myth or reality Rev. Bras. Obstet. Gynecol., 36: 143-5, 2014.

7- POON L.C., VOLPE N., MUTO B., YU C.K., SYNGELAKI A. and NICOLAIDES K.H.: Second-trimester uterine artery Doppler in the prediction of stillbirths. Fetal Diagn. Ther., 33: 28-35, 2013.

8- PAPAGEORGHIOU A.T., YU C.K., CICERO S., BOWER S. and NICOLAIDES K.H.: Secondtrimester uterine artery Doppler screening in unselected populations: A review. J. Matern. Fetal. Neonatal. Med., 12: 78-88, 2002.

9- BERKLEY E., CHAUHAN S.P. and ABUHAMAD A.: Doppler assessment of the fetus with intrauterine growth restriction. Am. J. Obstet. Gynecol., 206: 300-8, 2012.

10-FITZPATRICK K.E., SELLERS S., SPARK P., KURINCZUK J.J., BROCKLEHURST P. and KNIGHT M.: Incidence and risk factors for placenta accreta/increta/percreta in the UK: A national case-control study. PLOS One, 7 (12): e52893, 2012.

11- CHO H.Y., HWANG H.S., JUNG I., PARK Y.W., KWON J. and KIM Y.H.: Diagnosis of Placenta Accreta by Uterine Artery Doppler Velocimetry in Patients With Placenta Previa. Journal of Ultrasound in Medicine, 34.9: 1571$5,2015$.

12-ZAMANSKAYA T.A., BUSHTYREV A. V., BUSHTYREVA I.O., STASHKEVICH V.V. and ANTIMIROVA V.V.: The uterine and fetoplacental hemodynamics in pregnant women with placenta previa and placenta accreta. Obstetrics, Gynecology and Reproduction, 11 (3): 5-10, 2017.

13- USTA I.M., HOBEIKA E.M., MUSA A.A., GABRIEL G.E. and NASSAR A.H.: Placenta previa-accreta: Risk factors and complications. Am. J. Obstet. Gynecol., 193: 1045-9, 2005 


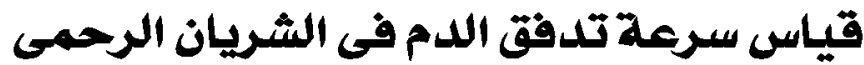

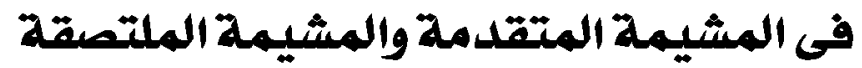

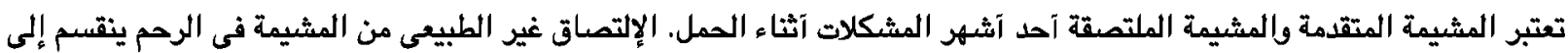

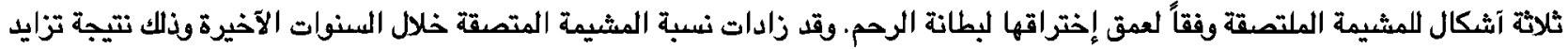

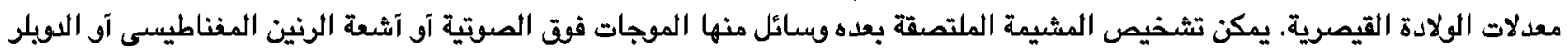

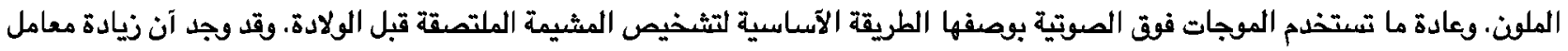

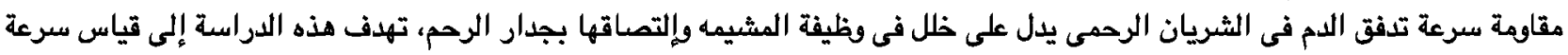

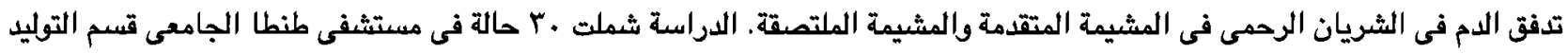

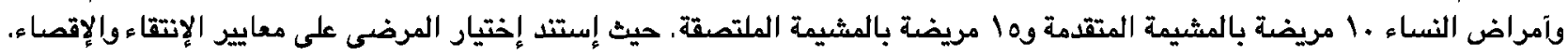

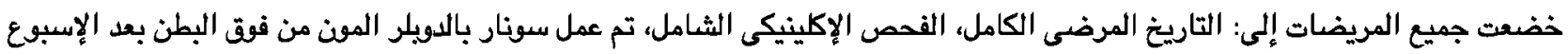

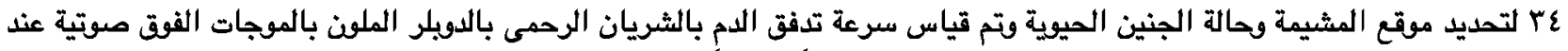

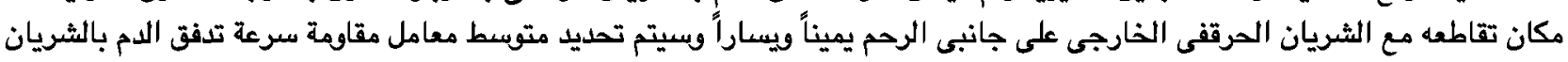

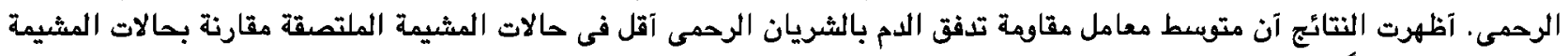

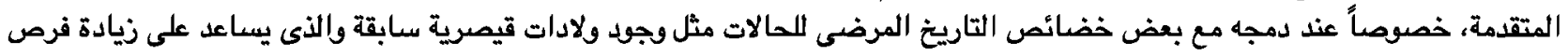

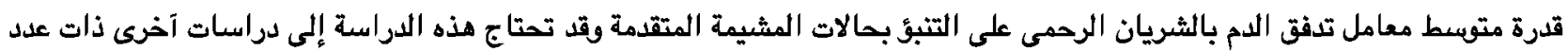

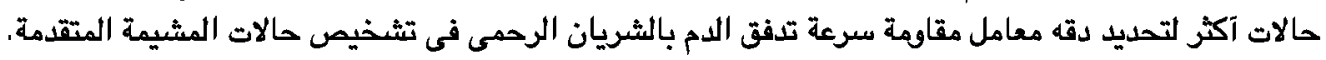

